\title{
A Coalition Formation Mechanism Based on Inter-Agent Trust Relationships
}

\author{
Silvia Breban \\ Julita Vassileva (author for correspondence) \\ University of Saskatchewan \\ 57 Campus Drive, S7N 5A9 \\ Saskatoon, SK, Canada \\ 1-306-966-2073 \\ jiv@cs.usask.ca
}

\begin{abstract}
We study long-term coalitions of customer and vendor software agents based on trust relationships among the agents. We propose a coalition formation mechanism designed at microscopic (agent) level and analyzed at both microscopic and macroscopic (system) levels. The evaluation compares agent strategies (individual vs. social) and analyzes the system behavior under different circumstances. The advantages of the mechanism are that it is simple, scales up well and does not require communication between the agents. It reduces the dynamic of the system and is beneficial for the individual agents (ensures higher individual gain).
\end{abstract}

Category Descriptors: I.2.11 [Distributed Artificial Intelligence]: Multi-agent Systems, Coherence and Coordination, Intelligent Agents

General Terms: Algorithms, Design, Economics, Experimentation

\section{INTRODUCTION}

There are only a few studies $[1,2,3]$ on coalition formation for the electronic marketplace. A limitation common to all these studies is that they model coalitions that last one transaction. Another limitation is that each agent in the system has to decide each time it wants to buy a product what coalition to join without memory of previous experiences. At the microscopic level, the search for suitable coalitions and the decision of what coalition to join is time and resource consuming. At the macroscopic level, forming and running new coalitions at each step is also computationally expensive. It leads to increased dynamics of the system (e.g. high variation in the number of coalitions existing in the system and in the size of each coalition) that is not desirable in a large-scale multi-agent system, since it brings instability and unpredictability.

Permission to make digital or hard copies of all or part of this work for personal or classroom use is granted without fee provided that copies are not made or distributed for profit or commercial advantage and that copies bear this notice and the full citation on the first page. To copy otherwise, or republish, to post on servers or to redistribute to lists, requires prior specific permission and/or a fee.

AAMAS '02, July 15-19, 2002, Bologna, Italy.

Copyright 2002 ACM 1-58113-480-0/02/0007 ...\$5.00.

\section{COALITION FORMATION}

We address an electronic marketplace composed of selfish agents with fixed roles of either customer or vendor. Agents have a long lifetime of repeated interactions with other agents in the system. They try at each moment to maximize their individual long-term utility functions. Agents may have different interests in items being traded and they may belong to different economic categories. The agents can form coalitions to increase their benefits.

Coalitions have a long lifetime once created. A coalition is automatically created when an agent wants to form a new coalition with another agent; it dissolves when it is composed of only two agents and one of them leaves the coalition. Agents in the same coalition agree with a specific discount for each transaction executed. Agents prefer to be part of the coalition in which they expect to have most future transactions. Coalitions are disjoint and the formed coalition structure is global knowledge.

The main reason for forming coalitions is to bring agents with compatible preferences closer, by nurturing vendor-customer relationships. This policy is motivated by the fact that establishing a friendly and trustworthy relationship with clients promises vendors more transactions for the long run and retention of customers, known as customer relationship management (CRM) [4]. For customers the discounts gained from transactions inside the same coalition are also profitable.

From the point of view of an individual agent - either customer or vendor - we see coalition formation as a decision problem: at each moment an agent faces a decision of whether to remain in the same status, form a new coalition, or leave the current coalition to join another one. The decision should maximize the agent's long-term utility function by taking into account the agent's global knowledge about the system and also its local knowledge about the other agents. This knowledge is stored in the form of trust relationships established with the other agents after sharing common experiences. In our context the trust of an agent in another agent is its belief that they will have satisfactory transactions in the future. It reflects the two agents having similar preferences and being trustworthy (i.e. deliver items with good quality and in time or pay in time). To represent these trust relationships in the agent's mind we use an existing model [5] for representing subjective trust with a numerical value from the interval $[-1,1]$ assigned to each trust relationship. 
The general scheme of our coalition formation mechanism is shown in Figure 1. It consists of two parts. The first one - the interaction between a vendor and a customer - starts with the agents negotiating on a price [6], it continues with a transaction, and it ends with an evaluation of the interaction and an update of the trust relationship by each of the agents. If the negotiation ends with rejection, the transaction is cancelled and the evaluation is negative. If the negotiation ends with agreement, the evaluation is positive. A discount is applied, if the two agents belong to the same coalition. The transaction is executed. In both cases, the new experience (positive or negative) is evaluated and the trust of the agents in each other is updated according to the model in [5].

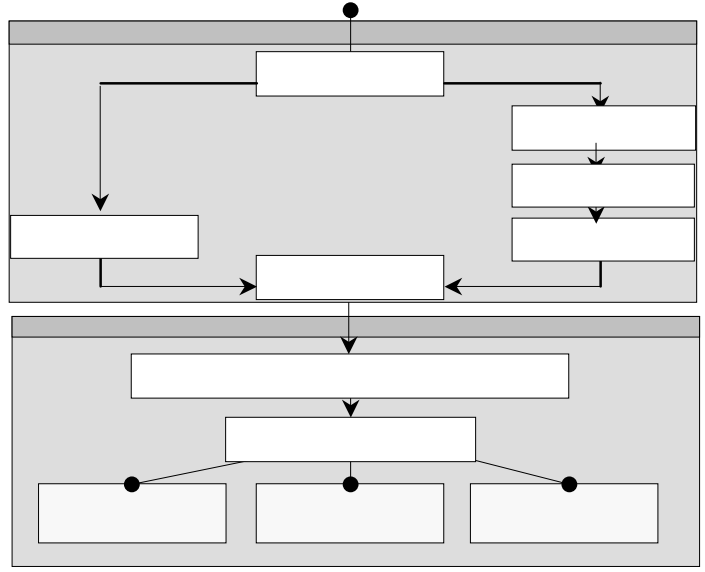

Figure 1: The coalition formation mechanism

In the second part of our general scheme the agent makes a decision about what coalition is most profitable and it performs an action that brings it into that coalition. The agent may prefer the coalition in which its most trusted agent is (individually oriented strategy) or the coalition in which it has the highest cumulative trust (socially oriented strategy) [7].

We performed both microscopic and macroscopic evaluations of the proposed coalition formation mechanism investigating three agent strategies and different setups. For the macroscopic analysis we investigated the number of coalitions in the system, the overall dynamics, and how these factors evolve in time. The evolution of the number of coalitions is relevant for reasons of predictability while the system dynamics (calculated as the sum of the number of coalitions visited by each agent) is important in establishing whether the system reaches an equilibrium state or not. For the microscopic evaluation we focused on the individual gains of the customer agents (calculated as the average of the sum of benefits obtained from all discounted transactions by each customer). The evaluation is based on a simulation with numbers of agents varying from 100 to 10,000 engaging in 1,000,000 interactions.

Our results $[7,8,9]$ showed that the number of coalitions has a predictable and controllable evolution over time. First it increases as the coalitions are formed and after a while it decreases since the coalitions start to merge. The number of coalitions has as an upper limit - the number of vendors and a lower limit - the number of preference categories of agents. The system dynamics increases over time. Stabilization is observed when the agents join the coalition with the highest number of trusted agents. In this case the individual gains of customers are also higher than in other cases.

We compared three agent strategies. The strategy where an agent prefers the coalition with the highest number of trusted agents proved to be the most beneficial for the system's predictability and dynamics as well as for the individual agents. The strategy where an agent prefers the coalition with the highest summative trust was found to be the worst, while the strategy where an agent prefers the coalition with the most trusted individual agent performed in between these two.

\section{CONCLUSIONS AND FUTURE WORK}

In this paper, we proposed and evaluated a coalition formation mechanism that takes into consideration trust relationships between agents. The mechanism extends the existing concept of temporary customer coalitions to long-term coalitions formed of both customers and vendors. We showed that our coalition formation mechanism brings stability to the system (in the number of coalitions and in the overall dynamics) and provides the customer agents increased benefits over time. The mechanism uses reduced communication between the agents that makes it scalable for large numbers of agents and interactions. Future work includes investigation of the proposed coalition formation mechanism under more realistic circumstances such as allowing more types of goods to be traded in the system and giving the agents the liberty to belong to more coalitions at a time.

\section{REFERENCES}

[1] M Tsvetovat, K. Sycara Customer Coalitions in the Electronic Marketplace Proceedings of the Fourth International Conference on Autonomous Agents, Barcelona, 2000, pp.263-264.

[2] K. Lermann, O. Shehory Coalition Formation for Large Scale Electronic Markets Proceedings of the Fourth International Conference on Multiagent Systems ICMAS'2000, Boston, July 2000, pp. $216-222$.

[3] J. Yamamoto, K. Sycara A Stable and Efficient Buyer Coalition Formation Scheme for E-marketplaces Proceedings of Autonomous Agents 2001, pp. 576-583.

[4] Customer Relationships Management http://www.crm-expo.com/

[5] C. Jonker, J. Treur Formal Analysis of Models for the Dynamics of Trust based on Experiences Autonomous Agents, Deception, Fraud and Trust in Agent Societies, Seattle 1999, pp. 81-94.

[6] C. Mudgal, J. Vassileva Bilateral Negotiation with Incomplete and Uncertain Information: A Decision-Theoretic Approach Using a Model of the Opponent Proceedings of the Workshop on Cooperative Information Agents (CIA IV), Boston, July 2000, pp. 107-118.

[7] S. Breban, J. Vassileva Using Inter-Agent Trust Relationships for Coalition Formation 15th Canadian Conference on A.I., Calgary, May 2002.

[8] S. Breban Long-Term Coalitions for the Electronic Marketplace. M.Sc. Thesis, University of Saskatchewan, February 2002. Available on-line from http://bistrica.usask.ca/madmuc/

[9] J. Vassileva, S. Breban, M. Horsch, Agent Reasoning Mechanism for Long-term Coalitions Based on Decision Making and Trust to appear in Computational Intelligence, Special Issue on Agent Technology for Electronic Issue. 